\title{
1 Comparative Analysis of Emerging B.1.1.7+E484K SARS-CoV-2
}

2 isolates from Pennsylvania

3

4 Ahmed M. Moustafa ${ }^{1,2}$, Colleen Bianco ${ }^{1}$, Lidiya Denu' ${ }^{1}$, Azad Ahmed ${ }^{3}$, Brandy Neide ${ }^{1}$,

5 John Everett ${ }^{4}$, Shantan Reddy ${ }^{4}$, Emilie Rabut ${ }^{5}$, Jasmine Deseignora ${ }^{5}$, Michael D.

6 Feldman $^{6}$, Kyle G. Rodino ${ }^{6}$, Frederic Bushman ${ }^{4}$, Rebecca M. Harris ${ }^{6,7}$, Josh Chang

7 Mell $^{3}$, Paul J. Planet ${ }^{1,7,8^{*}}$

1. Division of Pediatric Infectious Diseases, Children's Hospital of Philadelphia,

10 Philadelphia, PA 19104, USA.

2. Division of Gastroenterology, Hepatology, and Nutrition, Children's Hospital of

13 Philadelphia, Philadelphia, PA 19104, USA.

3. Department of Microbiology and Immunology, Center for Genomic Sciences, 
23 6. Department of Pathology and Laboratory Medicine, Perelman School of

24 Medicine, University of Pennsylvania, Philadelphia, PA 19104, USA.

25

26 7. Department of Pediatrics, Perelman College of Medicine, University of

27 Pennsylvania, Philadelphia, PA 19104, USA.

28

8. Sackler Institute for Comparative Genomics, American Museum of Natural

History, New York, NY 10024, USA.

31

32 Phone

33 PJP: +1 215-590-1169

$34 \quad{ }^{*}$ Corresponding Author

35 Emails

36 AMM: moustafaam@chop.edu

37 PJP: planetp@chop.edu 


\section{Abstract}

39 Rapid whole genome sequencing of SARS-CoV-2 has presented the ability to detect new emerging variants of concern in near real time. Here we report the genome of a

41 virus isolated in Pennsylvania in March 2021 that was identified as lineage B.1.1.7

42 (VOC-202012/01) that also harbors the E484K spike mutation, which has been shown

43 to promote "escape" from neutralizing antibodies in vitro. We compare this sequence to

44 the only 5 other B.1.1.7+E484K genomes from Pennsylvania, all of which were isolated

45 in mid March. Beginning in February 2021, only a small number $(n=60)$ of isolates with

46 this profile have been detected in the US, and only a total of 253 have been reported

47 globally (first in the UK in December 2020). Comparative genomics of all currently

48 available high coverage B.1.1.7+E484K genomes $(n=235)$ available on GISAID

49 suggested the existence of 7 distinct groups or clonal complexes (CC; as defined by

50 GNUVID) bearing the E484K mutation raising the possibility of 7 independent

51 acquisitions of the E484K spike mutation in each background. Phylogenetic analysis

52 suggested the presence of at least 3 distinct clades of B.1.1.7+E484K circulating in the

53 US, with the Pennsylvanian isolates belonging to two distinct clades. Increased genomic

54 surveillance will be crucial for detection of emerging variants of concern that can escape

55 natural and vaccine induced immunity. 
During the past six months of the pandemic several variants of concern (VOC), each represented by a constellation of specific mutations thought to enhance viral fitness,

59 have emerged in viral lineages from the UK (20I/501Y.V1; B.1.1.7), South Africa

60 (20H/501Y.V2; B.1.351), and Brazil (20J/501Y.V3; P.1). These lineages were

61 concerning due to likely increased transmission rates ${ }^{1-6}$. Two of these lineages, B.1.351

62 and P.1 were of specific concern because they harbor the mutation E484K, which has

63 been shown to enhance escape from neutralizing antibody inhibition in vitro ${ }^{7}$, and may

64 be associated with reduced efficacy of the vaccine ${ }^{8-11}$. In general, viruses from the

65 B.1.1.7 lineage do not harbor this mutation. However, in February 2021 Public Health

66 England (PHE) published a concerning report of eleven B.1.1.7 genomes that had

67 acquired the E484K spike mutation ${ }^{12}$.

68 Here we report a B.1.1.7 isolate with the E484K spike mutation isolated in

69 southeastern Pennsylvania (PA). Our laboratory at the Children's hospital of

70 Philadelphia performed sequencing on randomly selected isolates collected since

71 January 2021. Figure 1A shows the diversity of 114 randomly sequenced genomes.

72 Lineages B.1.1.7, B.1.429 (California), B.1.526 (New York) and R.1 (International

73 lineage with the E484K mutation) accounted for $69 \%$ of the sequenced genomes in

74 March. There was a massive increase in lineage B.1.1.7 from 2\% (1/47) in February to

$7542 \%$ in March (15/36). Interestingly, one B.1.1.7 isolate carried the E484K spike

76 mutation that is present in the South African and Brazilian lineages.

77 To better understand the relationship between this isolate and publicly available

78 SARS-CoV-2 genomes, we compared it to all available B.1.1.7+E484K high coverage

79 genomes available on $\operatorname{GISAID}^{13}(\mathrm{n}=235)$. Since the first report by PHE in February, a 
80 total of 253 B.1.1.7+E484K genomes have been uploaded to GISAID from England and

8114 other countries (Germany, France, Italy, Poland, Sweden, Ireland, Netherlands,

82 Portugal, Wales, Turkey, Slovakia, Austria, Czech Republic and USA) ${ }^{13}$ (as of

$8304 / 17 / 2021)$

84 A temporal plot of the number of B.1.1.7+E484K isolates collected between

85 December 2020 to March 2021 (2-week window) is shown in Figure 1B. The first

86 isolate of the 60 US isolates available on GISAID was collected on 02/06/2021 from

87 Oregon (OR). Isolates were also reported from 15 other states (New York, North

88 Carolina, Connecticut, Georgia, New Jersey, Maryland, Florida, West Virginia,

89 California, Pennsylvania, Michigan, Texas, Massachusetts, Washington, and Colorado).

90 Of these isolates $48 \%$ were from Florida $(n=17)$ and New York $(n=12)$ and $28 \%$ were

91 from New Jersey $(n=7)$, California $(n=4)$ and Pennsylvania $(n=6)$. Two isolates were

92 from Oregon (OR), Connecticut (CT), Maryland (MD), and single isolates are recorded

93 from Georgia (GA), Texas (TX), Massachusetts (MA), Washington (WA), Colorado

94 (CO), West Virginia (WV), Michigan (MI), and North Carolina (NC). The number of US

95 isolates in March ( $n=47$ including the PA isolates) was nearly 6 times the number of the

96 isolates reported in February. This increase raises the concern that more

97 B.1.1.7+E484K sequences may be emerging even as herd immunity increases by

98 natural immunity and vaccines.

99 Although all 236 genomes were typed as B.1.1.7 using Pangolin ${ }^{14}$, a more granular

100 view using our typing tool "GNUVID"15 shows that they belong to 7 different clonal

101 complexes (CCs 45062, 46649, 49676, 57630, 58534, 62415 and 67441) (Figure 1C

102 and Supplementary Table 1). In the GNUVID typing system, these correspond to 7 of 
$10310 \mathrm{CCs}$ in the B.1.1.7 lineage. For each of these CCs, representative sequences

104 without the E484K mutation have been circulating since at least November 2020,

105 predating the first E484K in each CC. This raises the possibility that the E484K mutation

106 was acquired independently in each of these CCs in independent events.

108 US isolates are found in at least 3 different clades. The genome presented here falls in 109 a well-supported clade of 28 isolates, 6 of which were from the US (CT, FL, OR, PA and

110 NY), 18 from Sweden, 2 from Poland and 1 from Germany (Figure 2A). The only other

1114 isolates reported from PA, were in a large clade containing the majority of US

112 genomes, and were located in a well-supported subclade with genomes from the nearby 113 state of West Virginia.

Analysis of SNPs in the 236 isolates compared to the reference MN908947.3 ${ }^{16}$

115 (Figure 2B and Supplementary Figure 1) showed that the isolate presented here had

$11612 / 17$ of the B.1.1.7 defining SNPs (Supplementary Table 2), while the other

117 Pennsylvanian isolate in the same clade had $17 / 17$ of the SNPs. It also shared with 9

118 other US isolates a stop mutation (A28095T) in ORF8 (Figure 2B).

120 detected in PA that harbor the E484K spike mutation, a mutation that could be

121 associated with reduced efficacy of both vaccine-induced and natural immunity. Our

122 analysis suggests that multiple lineages of B.1.1.7+E484K are circulating in the US, and

123 that these lineages may have acquired E484K independently.

\section{Methods}


A nasopharyngeal swab sample that had residual volume after initial laboratory

127 processing, positive PCR testing for SARS-CoV-2, was obtained for this study. RNA

128 was extracted from nasopharyngeal swab samples using QIAamp Viral RNA Mini

129 (Qiagen). Whole genome sequencing was done by The Genomics Core Facility at

130 Drexel University. Briefly, WGS of extracted viral RNA was performed as previously

131 described using Paragon Genomics CleanPlex SARS-CoV-2 Research and

132 Surveillance NGS Panel ${ }^{17,18}$. Libraries were quantified using the Qubit dsDNA HS (High

133 Sensitivity) Assay Kit (Invitrogen) with the Qubit Fluorometer (Invitrogen). Library quality

134 was assessed using Agilent High Sensitivity DNA Kit and the 2100 Bioanalyzer

135 instrument (Agilent). Libraries were then normalized to 5nM and pooled in equimolar

136 concentrations. The resulting pool was quantified again using the Qubit dsDNA HS

137 (High Sensitivity) Assay Kit (Invitrogen) and diluted to a final concentration of 4nM;

138 libraries were denatured and diluted according to Illumina protocols and loaded on the

139 MiSeq at 10pM. Paired-end and dual-indexed 2x150bp sequencing was done using

140 MiSeq Reagent Kits v3 (300 cycles). Sequences were demultiplexed and basecalls

141 were converted to FASTQ using bcl2fastq2 v2.20. The FASTQ reads were then

142 processed to consensus sequence and variants were identified using the ncov2019-

143 artic-nf pipeline (https://github.com/connor-lab/ncov2019-artic-nf). Briefly, the pipeline

144 uses iVar $^{19}$ for primer trimming and consensus sequence making (options: --

145 ivarFreqThreshold 0.75). A bed file for the Paragon kit primers was used in the pipeline.

146 All 253 SARS-CoV-2 genomes that were assigned to Pango lineage ${ }^{14}$ B.1.1.7

147 and possessing the E484K spike mutation (including the study isolate CHOP_204) were

148 downloaded from GISAID ${ }^{13}$ on 04/17/2021. An acknowledgement table of the submitting 
149 laboratories providing the SARS-CoV-2 genomes used in this study is in Supplemental

150 Table 3. Seventeen sequences were excluded for lower coverage $(>5 \%$ Ns) $(n=14)$

151 and missing collection date $(n=3)$. All the high coverage SARS-CoV-2 genomes $(n=236)$

152 were assigned a clonal complex using the GNUVID v2.2 database (version January $6^{\text {th }}$

$1532021)^{15}$. Temporal plots were plotted in GraphPad Prism v7.0a.

154 To show the relationship amongst the genomes of the 236 isolates, a maximum

155 likelihood tree was constructed. Briefly, consensus SARS-CoV-2 sequences for the 236

156 isolates were aligned to MN908947. $3^{16}$ using MAFFT's FFT-NS-2 algorithm ${ }^{20}$ (options:

157 --add --keeplength)). The 5' and 3' untranslated regions were masked in the alignment

158 file using a custom script. A maximum likelihood tree using IQ-TREE $2^{21}$ was then

159 estimated using the $\mathrm{GTR}+\mathrm{F}+\mathrm{I}$ model of nucleotide substitution ${ }^{22}$, default heuristic search

160 options, and ultrafast bootstrapping with 1000 replicates $^{23}$. The tree was rooted to

161 MN908947.3. The snipit tool was then used to summarize the SNPs in the 236 isolates

162 relative to MN908947.3 (https://github.com/aineniamh/snipit).

163 The sample was obtained by as part of routine clinical care, solely for non-

164 research purposes, carrying minimal risk, and were therefore granted a waiver of

165 informed consent as reviewed under protocol number under IRB 21-018478.

167 Availability of data and material

168 The sequence has been uploaded to GISAID with accession number

169 EPI_ISL_1629709.

171 Conflict of interest 
172 The authors declare that they have no competing interests.

173

\section{Acknowledgements}

175 We would like to thank the Global Initiative on Sharing All Influenza Data (GISAID) and

176 thousands of contributing laboratories for making the genomes publicly available. A full

177 acknowledgements table is available in Supplementary Table 3. We would like to

178 acknowledge the staff members of the Drexel Genomics Core Facility at the Drexel

179 University College of Medicine for processing and sequencing the isolates. P.J.P and

180 A.M.M are supported by 1R01Al137526-01 and 1R21Al144561-01A1 (A.M.M. and

181 P.J.P.), and R01NR015639 (P.J.P.). 


\section{Figure Legends}

\section{Figure 1. Diversity of SARS-CoV-2 in Philadelphia and global diversity of}

sequenced B.1.1.7+E484K genomes. A. Stacked bar plot showing the diversity of

187 random genomes sequenced by our laboratory at Children's Hospital of Philadelphia

188 during January, February and March 2021. Ten lineages that were represented by only

189 one genome (B.1.1, B.1.1.106, B.1.1.129, B.1.1.197, B.1.1.281, B.1.1.296, B.1.119,

was counted with the parent B.1.526 for easier visualization. B. Bar plot showing number of GISAID genomes $(n=250)$ that are 20I/501Y.V1 and have the E484K spike

194 GNUVID. Bar plot showing relative abundance of circulating clonal complexes (CC) for

196 isolates belong to 7 different CCs. Isolate EPI_ISL_1385215 was not assigned to any of 197 the 7 CCs (CC255). Fourteen isolates were excluded from the plot as they had $>5 \%$

198 nucleotides designated "N" in the sequence.

199 Figure 2. SNP-based Phylogeny and variations of the B.1.1.7+E484K isolates. A.

200 Maximum likelihood tree of the B.1.1.7+E484K isolates. US isolates are in red. For the

201 CHOP_204 isolate the alternative allele was called as consensus if its frequency was at

202 least 0.75. The tree was rooted with MN908947.3. Bootstrap values are shown on the

203 branches. B. SNP patterns in the 53 US isolates compared to MN908947.3. SNP

204 variations in the 236 isolates are shown in Supplementary Figure 1. Mutations identified

205 in CHOP_204 are available in Supplementary Table 2. Seven US isolates were 206 excluded from the plot as they had $>5 \%$ nucleotides designated "N" in the sequence. 
bioRxiv preprint doi: https://doi.org/10.1101/2021.04.21.440801; this version posted April 21, 2021. The copyright holder for this preprint (which

was not certified by peer review) is the author/funder, who has granted bioRxiv a license to display the preprint in perpetuity. It is made available under aCC-BY-NC-ND 4.0 International license.

207 An acknowledgement table of the submitting laboratories providing the SARS-CoV-2

208 genomes used in this study is in Supplemental Table 3.

209 
bioRxiv preprint doi: https://doi.org/10.1101/2021.04.21.440801; this version posted April 21, 2021. The copyright holder for this preprint (which was not certified by peer review) is the author/funder, who has granted bioRxiv a license to display the preprint in perpetuity. It is made available under aCC-BY-NC-ND 4.0 International license.

\section{Figure 1}

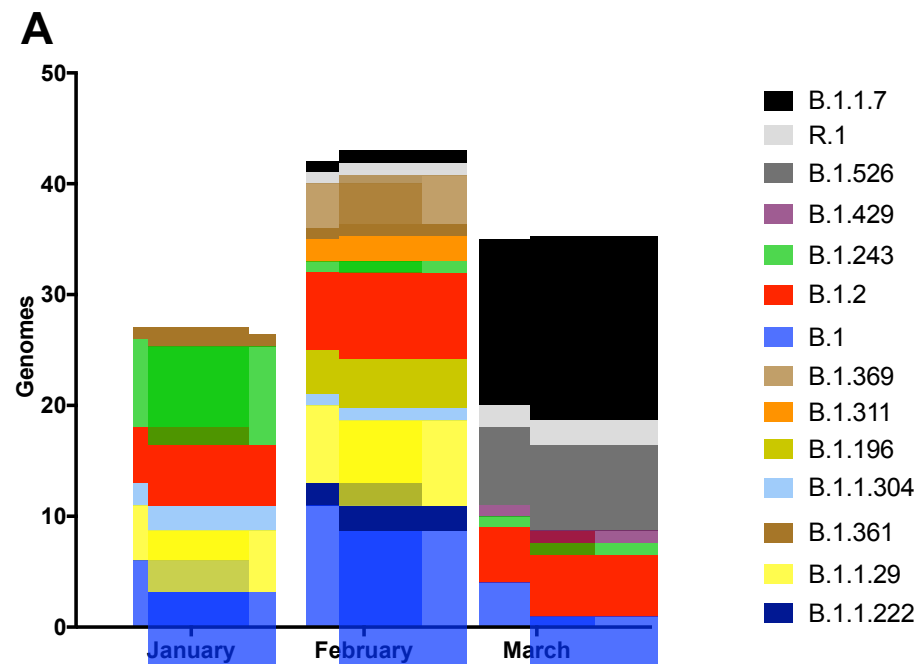

B
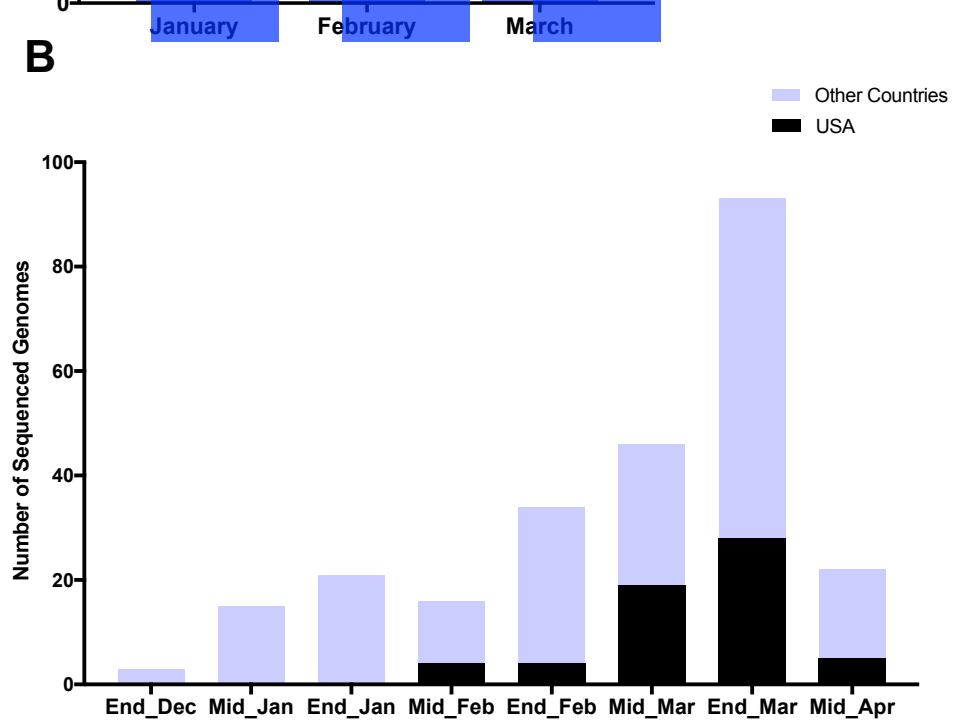

\section{C}

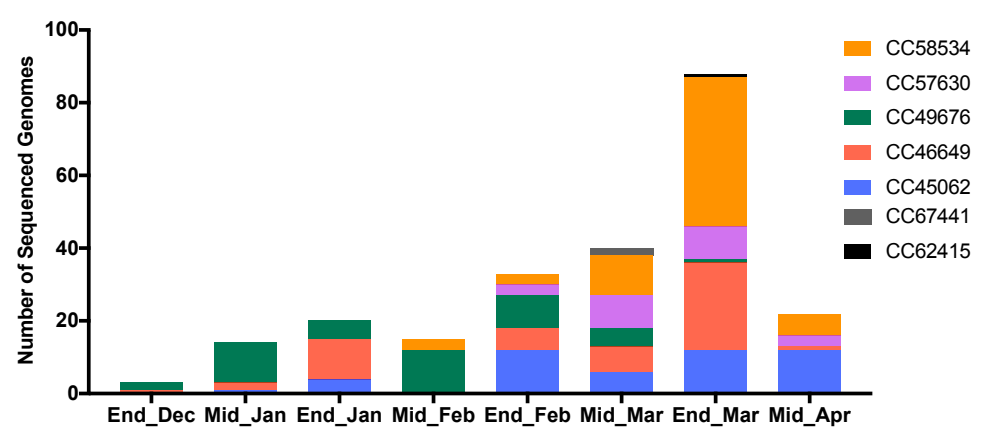


bioRxiv preprint doi: https://doi.org/10.1101/2021.04.21.440801; this version posted April 21, 2021. The copyright holder for this preprint (which was not certified by peer review) is the author/funder, who has granted bioRxiv a license to display the preprint in perpetuity. It is made available under aCC-BY-NC-ND 4.0 International license.

\section{Figure 2}

A

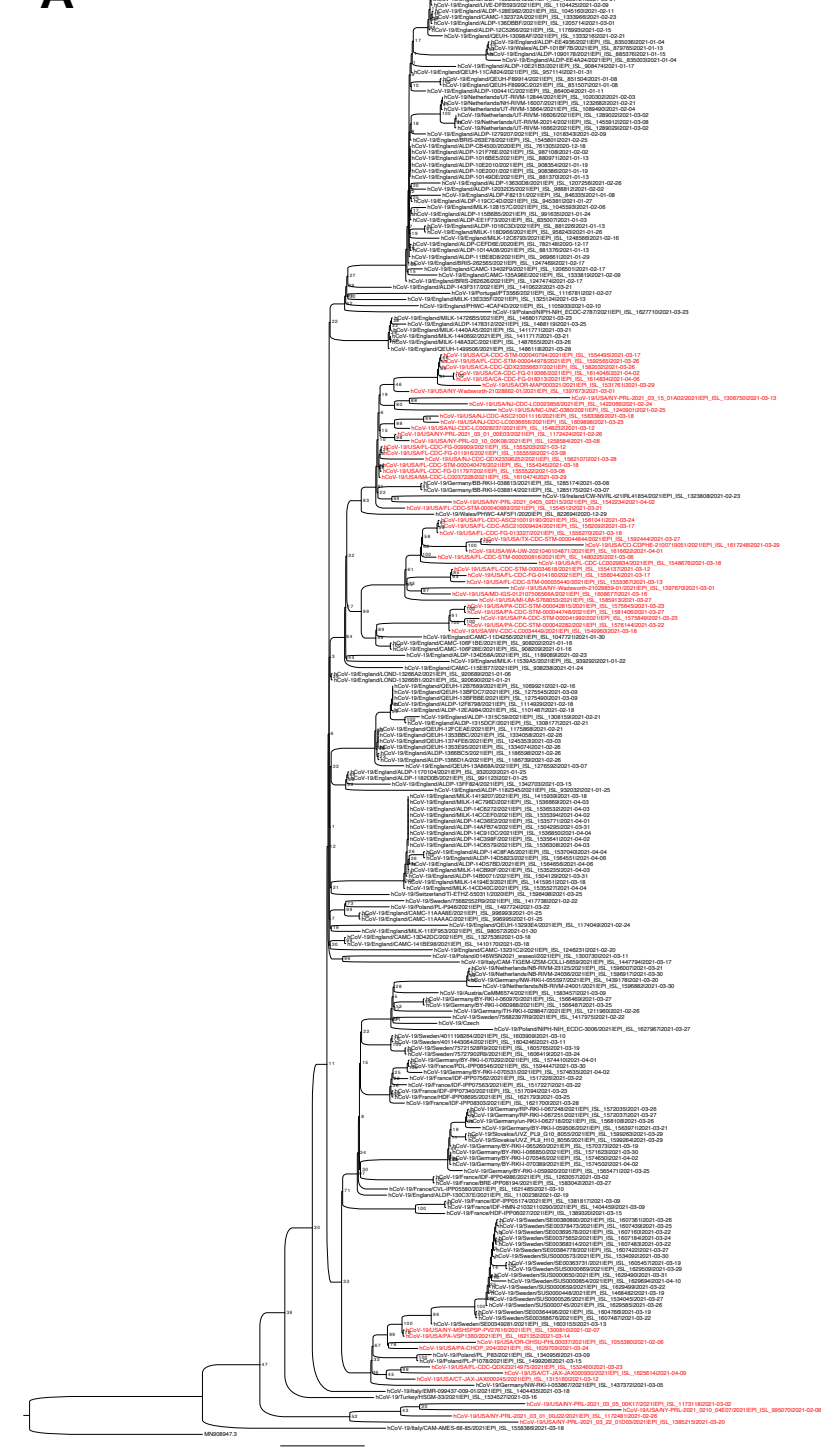

B

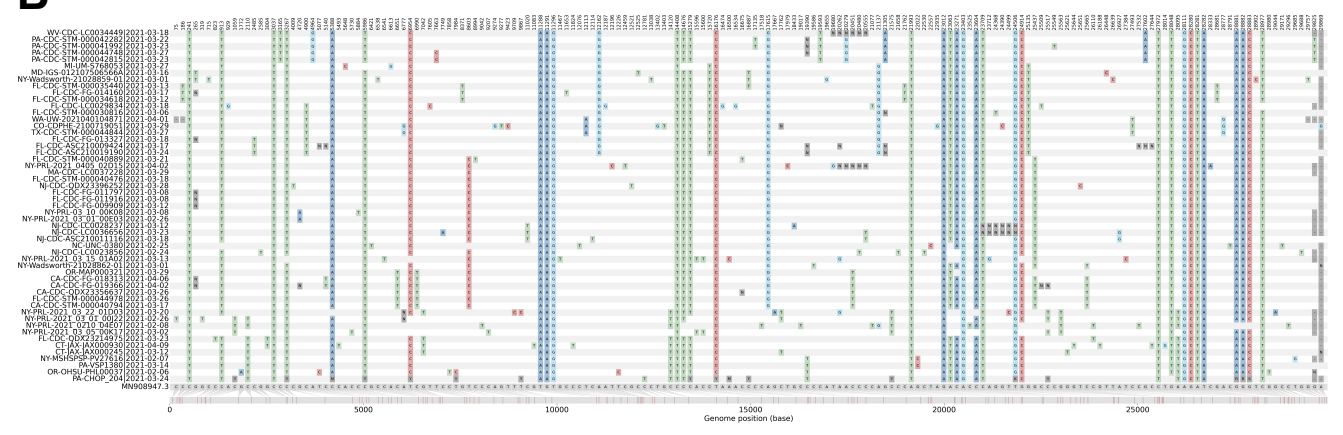




\section{Supplementary Table 1. Excel Sheet of GNUVID results for the 236 isolates.}

215 Supplementary Table 2. Mutations and deletions in CHOP_204 compared to

216 MN908947.3.

\begin{tabular}{|c|c|c|c|}
\hline Mutation & Protein & AA change & Frequency \\
\hline $\mathrm{C} 241 \mathrm{~T}$ & - & - & 1 \\
\hline C913T & ORF1ab & synonymous & 0.92 \\
\hline C1059T & ORF1ab & T265I & 0.33 \\
\hline C2110T & ORF1ab & synonymous & 0.98 \\
\hline C3037T & ORF1ab & synonymous & 1 \\
\hline C3267T & ORF1ab & T1001I & 0.64 \\
\hline C4320T & ORF1ab & synonymous & 0.38 \\
\hline C5388A & ORF1ab & A1708D & 0.65 \\
\hline C5986T & ORF1ab & synonymous & 0.62 \\
\hline T6954C & ORF1ab & I2230T & 0.74 \\
\hline T7984C & ORF1ab & synonymous & 0.65 \\
\hline T9867C & ORF1ab & L3201P & 0.33 \\
\hline 11288 (del-9) & ORF1ab & SGF3675-77 deletion & 0.99 \\
\hline $\mathrm{C} 12781 \mathrm{~T}$ & ORF1ab & synonymous & 0.96 \\
\hline C14120T & ORF1ab & Q4619* & 0.95 \\
\hline C14408T & ORF1ab & synonymous & 1 \\
\hline C14676T & ORF1ab & P4804L & 0.96 \\
\hline C15279T & ORF1ab & T5005I & 0.66 \\
\hline T16176C & ORF1ab & L5304P & 0.72 \\
\hline A16500C & ORF1ab & K5412T & 0.30 \\
\hline C16887T & ORF1ab & synonymous & 0.31 \\
\hline C19390T & ORF1ab & synonymous & 1 \\
\hline $\mathrm{C} 21575 \mathrm{~T}$ & $S$ & L5F & 0.35 \\
\hline 21765 (del6) & $\mathrm{S}$ & HV69-70 deletion & 0.99 \\
\hline 21991 (del3) & $\mathrm{S}$ & Y144 deletion & 0.98 \\
\hline G23012A & $\mathrm{S}$ & E484K & 0.77 \\
\hline A23063T & $\mathrm{S}$ & N501Y & 0.95 \\
\hline C23271A & $\mathrm{S}$ & A570D & 1 \\
\hline A23403G & $S$ & D614G & 1 \\
\hline
\end{tabular}




\begin{tabular}{cccc}
\hline C23604A & S & P681H & 0.98 \\
C23664T & S & A701V & 0.41 \\
C23709T & $\mathrm{S}$ & T716I & 0.99 \\
T24506G & $\mathrm{S}$ & S982A & 0.55 \\
\hline G24914C & $\mathrm{S}$ & $\mathrm{D} 1118 \mathrm{H}$ & 0.94 \\
\hline C25517T & ORF3a & P42L & 0.36 \\
\hline C27972T & ORF8 & Q27* & 0.93 \\
\hline A28095T & ORF8 & K68* & 0.93 \\
\hline A28111G & ORF8 & Y73C & 0.96 \\
\hline A28271 (del1) & - & deletion & 0.97 \\
\hline GAT28280CTA & $\mathrm{N}$ & D3L & 0.97 \\
\hline C28869T & $\mathrm{N}$ & P199L & 0.38 \\
\hline GG $28881 \mathrm{AAC}$ & $\mathrm{N}$ & R203K, G204R & 0.55 \\
\hline C28977T & $\mathrm{N}$ & S235F & 0.88 \\
\hline C29137T & $\mathrm{N}$ & synonymous & 0.54
\end{tabular}

\section{Supplementary Table 3. GISAID Acknowledgement Table.}


bioRxiv preprint doi: https://doi.org/10.1101/2021.04.21.440801; this version posted April 21, 2021. The copyright holder for this preprint (which

was not certified by peer review) is the author/funder, who has granted bioRxiv a license to display the preprint in perpetuity. It is made available under aCC-BY-NC-ND 4.0 International license.

\section{Supplementary Figure 1. SNP variations in all available 20I/501Y.V1+E484K}

230 isolates.

231

232 


\section{References}

1 Davies, N. G. et al. Estimated transmissibility and impact of SARS-CoV-2 lineage B.1.1.7 in England. Science, eabg3055, doi:10.1126/science.abg3055 (2021). syndrome-related coronavirus 2 (SARS-CoV-2) lineage with multiple spike mutations in South Africa. medRxiv, 2020.2012.2021.20248640, doi:10.1101/2020.12.21.20248640 (2020).

3 Faria, N. R. et al. Genomics and epidemiology of a novel SARS-CoV-2 lineage in Manaus,
Brazil. medRxiv, 2021.2002.2026.21252554, doi:10.1101/2021.02.26.21252554 (2021).

4 Rambaut, A., et al. Preliminary genomic characterisation of an emergent SARS-CoV-2 (2020).

5 Chen, R. E. et al. Resistance of SARS-CoV-2 variants to neutralization by monoclonal and serum-derived polyclonal antibodies. Nature Medicine, doi:10.1038/s41591-021-01294w (2021).

6 Volz, E. et al. Transmission of SARS-CoV-2 Lineage B.1.1.7 in England: Insights from linking epidemiological and genetic data. medRxiv, 2020.2012.2030.20249034, doi:10.1101/2020.12.30.20249034 (2021).

7 Weisblum, Y. et al. Escape from neutralizing antibodies by SARS-CoV-2 spike protein variants. elife 9, e61312, doi:10.7554/eLife.61312 (2020).

8 Zhou, D. et al. Evidence of escape of SARS-CoV-2 variant B.1.351 from natural and vaccine-induced sera. Cell, doi:10.1016/j.cell.2021.02.037.

2569 Garcia-Beltran, W. F. et al. Multiple SARS-CoV-2 variants escape neutralization by vaccine-induced humoral immunity. Cell, doi:10.1016/j.cell.2021.03.013 (2021). Wu, K. et al. Serum Neutralizing Activity Elicited by mRNA-1273 Vaccine. N Engl J Med, doi:10.1056/NEJMc2102179 (2021).

Collier, D. A. et al. Sensitivity of SARS-CoV-2 B.1.1.7 to mRNA vaccine-elicited antibodies. Nature, doi:10.1038/s41586-021-03412-7 (2021).

1 Public Health England. Investigation of novel SARS-CoV-2 variant: Variant of Concern 202012/01 (Technical briefing 5). https://assets.publishing.service.gov.uk/government/uploads/system/uploads/attachme nt data/file/959426/Variant of Concern VOC 20201201 Technical Briefing 5.pdf (2021).

13 Shu, Y. \& McCauley, J. GISAID: Global initiative on sharing all influenza data - from vision to reality. Euro Surveill 22, doi:10.2807/1560-7917.ES.2017.22.13.30494 (2017).

14 Rambaut, A. et al. A dynamic nomenclature proposal for SARS-CoV-2 lineages to assist genomic epidemiology. Nat Microbiol, doi:10.1038/s41564-020-0770-5 (2020).

15 Moustafa, A. M. \& Planet, P. J. Emerging SARS-CoV-2 diversity revealed by rapid whole genome sequence typing. bioRxiv, doi:10.1101/2020.12.28.424582 (2020).

16 Wu, F. et al. A new coronavirus associated with human respiratory disease in China. Nature 579, 265-269, doi:10.1038/s41586-020-2008-3 (2020). 
$27517 \quad \mathrm{Li}, \mathrm{C}$. et al. Highly sensitive and full-genome interrogation of SARS-CoV-2 using multiplexed PCR enrichment followed by next-generation sequencing. bioRxiv, 2020.2003.2012.988246, doi:10.1101/2020.03.12.988246 (2020).

27818 Pandey, U. et al. High Prevalence of SARS-CoV-2 Genetic Variation and D614G Mutation in Pediatric Patients with COVID-19. Open Forum Infectious Diseases (2020 (In Press)). measuring intrahost virus diversity using PrimalSeq and iVar. Genome Biol 20, 8, doi:10.1186/s13059-018-1618-7 (2019).

20 Katoh, K., Misawa, K., Kuma, K. \& Miyata, T. MAFFT: a novel method for rapid multiple sequence alignment based on fast Fourier transform. Nucleic Acids Res 30, 3059-3066, doi:10.1093/nar/gkf436 (2002). doi:10.1093/molbev/msaa015 (2020).

29123 Hoang, D. T., Chernomor, O., von Haeseler, A., Minh, B. Q. \& Vinh, L. S. UFBoot2: Improving the Ultrafast Bootstrap Approximation. Mol Biol Evol 35, 518-522, doi:10.1093/molbev/msx281 (2018). 